\title{
The Development of the Power Management Circuit of Vibrational Energy Harvesting Devices
}

\author{
CHEN Yushi, FANG Yuming, DING Liqun, YANG Mengyuan, Niu Fei \\ Department of microelectronics, Nanjing University of Posts and Telecommunications, Nanjing, \\ 210023, China \\ Email: 1078185972@qq.com fangym@njupt.edu.cn
}

Keywords: Vibration Energy Harvesting; Power Management Circuits; MEMS Technology

\begin{abstract}
The development of micro devices is mainly restricted by chemical battery. Scientists have proposed the method to collect energy from the surroundings and convert it into electrical energy. This article summarizes different energy management circuit modules systematically and sums up the circuit structures. The advantages of different circuits are compared in detail to provide reference for designing a more reasonable circuit has higher output voltage and energy harvesting efficiency. This paper can provide reference for the related researchers.
\end{abstract}

\section{Introduction}

In recent years, with the rapid development of integrated circuits and MEMS technology, low power electronic equipments have been widely applied. However, the micro devices are mainly restricted by chemical battery. Therefore, energy harvesting device is an effective way to solve the problem of power supply. At present, there are three kinds of energy source: electromagnetic radiation, thermal energy and mechanical energy. Three kinds of energy harvesting methods based on mechanical vibration are piezoelectric, electrostatic and electromagnetic. The energy provided by environmental vibration is relatively weak and irregular, so the energy acquisition module cannot directly to provide sufficient and stable electricity to system. Therefore, we need to design suitable power management circuits to realize AC/DC conversion to provide stable energy supply.

\section{Power supply management circuit for energy device}

With the continuous progress of the research, a variety of energy structures have been proposed. The energy provided by environmental vibration is relatively weak and irregular, so we need to do electrical energy conversion to provide stable power to devices. As a result, we need to pay attention to the energy harvesting power management circuits [1] [2].Energy storage module is an important part of energy harvesting system. How to realize efficiently electrical energy conversion is the main direction of current research. The structure of the energy harvesting devices is shown in figure 1.

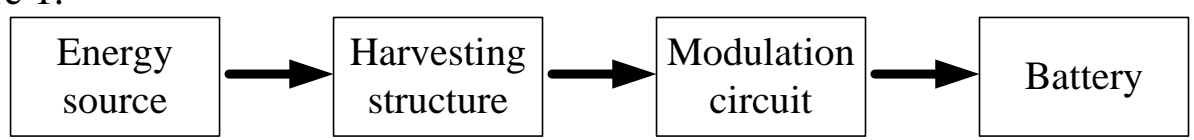

Figure 1 Structure of the energy harvesting devices

\section{Traditional energy acquisition circuit}

Traditional energy acquisition circuit includes a full bridge rectifier and a filter capacitor, the circuit is essentially an AC - DC conversion circuit. The role of the full-bridge rectifier is to convert ac voltage into a dc voltage. The filtering capacitor must be large enough to ensure the stable output voltage. As shown in figure 2 for simple RC bridge rectifier filter circuit. 


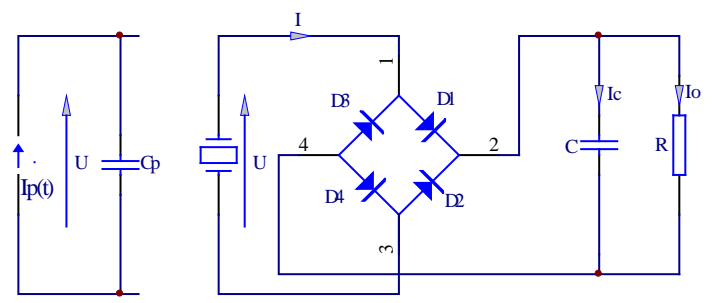

Figure 2 Traditional energy acquisition circuit

Piezoelectric energy harvesting structure as shown in figure 2, the piezoelectric vibrator is a capacitive device. It can be equivalent to a sinusoidal current source Ip(t) and internal capacitance $\mathrm{Cp}$ in parallel. The extreme value of current varies with the piezoelectric smart vibration level, the stronger the vibration, the larger the Ip. It is believed that it has nothing to do with the external load. The current frequency is equal to the vibration frequency.

Its working principle is shown in following. When voltage rises gradually, diode D1, D4 are on, D2, D3 are off, current direction is indicated by the arrows in figure 2. Because of the low resistance of power resistance and diode, the charging time is very short. Then the voltage of the $\mathrm{C}$ quickly rises to the peak. The charging process ends when voltage begins to decline after reaching the peak. Four diodes cut off due to the increase of the voltage at the ends of the capacitance $\mathrm{C}$. Then the capacitance $\mathrm{C}$ discharges to maintain the current of $\mathrm{R}$. The output waveform will be smooth.

Yuan Jiangbo[3] with the traditional energy collection circuit of piezoelectric cantilever beam single crystal generator has carried on the experimental study. When the excitation frequency of 57 $\mathrm{Hz}$, exciting force peak of $0.1 \mathrm{~N}$, the load of $10 \mathrm{k} \Omega$, the optimal capacity of the circuit is $1.18 \mathrm{mw}$. Bai Fengxian, Bao huayu et al of Dalian university of technology [4] in order to facilitate analysis of the electrical characteristics of piezoelectric vibration energy harvesting system, put forward to the method of equivalent for RC circuit based on the equivalent principle of piezoelectric energy gathering system.

\section{Synchronization charge extraction circuit}

The method of synchronous charge extraction circuit is to transfer the accumulated charge of the piezoelectric vibrator to the energy storage unit periodically. As shown in figure 3, its working principle is: when the displacement of piezoelectric vibrator reaches the maximum or minimum, its voltage at the ends is largest, the charge quickly transfers to the inductance L. The switch S shuts off after the completion of the transfer. Then energy is transferred through the $\mathrm{D}$ to the energy storage unit. Compared with traditional energy collection circuit, the efficiency of synchronous charge extraction circuit is high. Also the output is not easily affected by load change. The essence of which is a Buck/Boost converter.

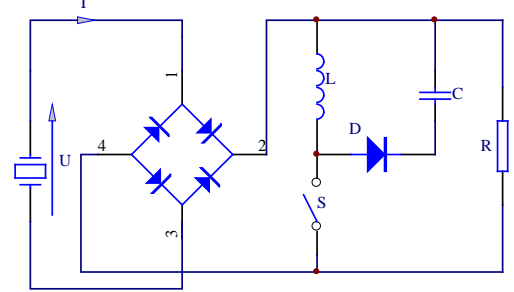

Figure 3 Synchronization charge extraction circuit

Lefeuvre [5] did related research about synchronous charge extraction circuit. Experimental tests show that the output power of synchronous extraction circuit energy charge collection has nothing to do with load size. The output of the average power of conventional energy harvesting circuit is four times that of the maximum power. The disadvantage of this circuit is that it is difficult to control the switch.

\section{Synchronous switch inductance circuit}

\section{Parallel synchronous switch inductance circuit}

Parallel synchronous switch inductance circuit is shown in figure 4. The switch $S$ is off in most of the time. When the vibration displacement of piezoelectric vibrator reaches extreme value, the switch circuit is on. Inductance $\mathrm{L}$ and internal capacitance of a piezoelectric crystal oscillator form a 
$\mathrm{LC}_{\mathrm{p}}$. It is purposed to achieve rapid reverse voltage at the ends of the piezoelectric crystal. The frequency of the oscillator is far higher than that of the vibration piezoelectric oscillator. It can assume that the voltage at the ends of the piezoelectric vibrator can turn in moment. We assume that at the maximum displacement, the top plate for the positive charge of piezoelectric vibrator, the bottom plate for the negative charge. Then the charge of two plates exchanges under the effect of the oscillator which avoids the drawback that the positive charge of the plate will decrease in the process. With the continuous increase of oscillator displacement, voltage at the ends of the piezoelectric vibrator increases constantly. It begins to provide power when the rectifier bridge conducts.

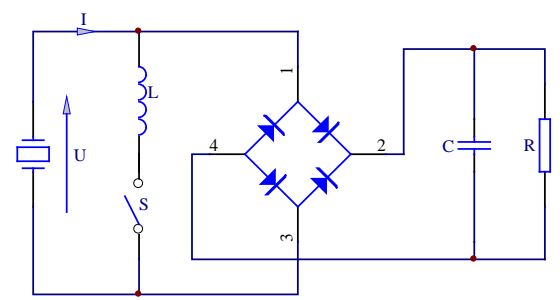

Figure 4 Parallel synchronous switch inductance circuit

Lefeuvre [6] has carried on the theoretical and experimental study on parallel synchronous switch inductance circuit. The results show that compared with the traditional energy collection circuit, the energy collection efficiency of the circuit can be increased by $400 \%$.

Series synchronous switch inductance circuit

Series synchronous switch inductance circuit and parallel synchronous switch inductance circuit are similar in structure. As shown in figure 5 . The difference is that the inductance $\mathrm{L}$ and switch $\mathrm{S}$ is connected in series between the piezoelectric element and the rectifier bridge.

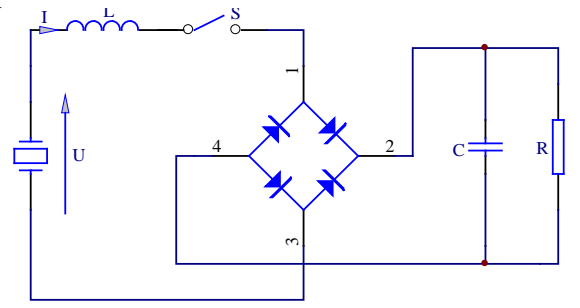

Figure 5 Series synchronous switch inductance circuit

Wang Hairen , Hu Hongping et al. [7]achieved SSHI process through the experiment, and verified the existence of the optimal rectifier voltage which makes piezoelectric energy harvester achieve the biggest output.

\section{DC - DC circuit}

\section{Buck DC - DC energy collection circuit}

Series switching voltage regulator circuit is also called buck circuit. When the switch is closed, $\mathrm{D}$ is cut-off, inductance $\mathrm{L}$ stores energy and capacitance $\mathrm{C}$ charges. When switch on, inductance $\mathrm{L}$ releases energy, meanwhile capacitance $C$ discharges. At the same time the direction of the electric current on the load resistance $\mathrm{R}$ is constant.

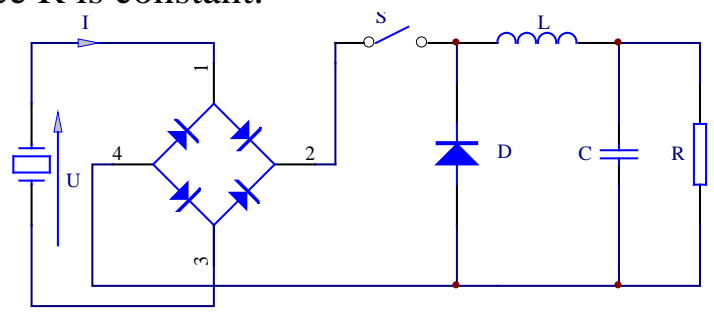

Figure 6 DC - DC energy collection circuit

Lesieutre [8] has carried on the related research on the circuit. The optimal duty ratio of energy collection circuit is $2.8 \%$, the efficiency reached $70 \%$. When exciting force is used on the piezoelectric vibration energy harvesting device and supplies a voltage of $48 \mathrm{v}$, the energy collected by the circuit is two times more than a traditional energy collected.

Boost type DC - DC energy collection circuit

Parallel switching voltage regulator circuit is also called boost circuit. This kind circuit is shown 
in figure 7. When the switch on, L stores energy, D is cut-off and filter capacitance C discharges to load resistance. When the switch is closed, L generates induced electromotive force, the same as the V. Two kinds of voltage combined by diode capacitance charges, regardless of the state of the switch tube and diode the current direction is always the same. When $\mathrm{L}$ is large enough, the voltage rises. When $C$ is large enough, the output voltage ripple can be small enough. When the cycle of the switch tube is constant, the higher duty ratio, the greater the output voltage will be.

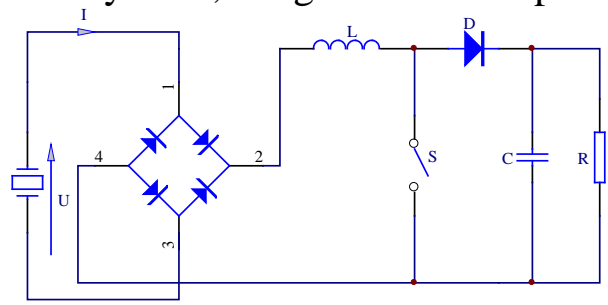

Figure 7 Booster type DC - DC energy collection circuit

Wahab, Shafii a. Sawal h. Md. Ali [9] and others designed boost circuit and simulated the parameters, and determine the effect of the passive components of booster circuit on output voltage. From the results of the simulation of induced resistance, the rise and fall of the switch time, the method can overcome the problem that the input voltage of low frequency vibration energy harvesters is low. Reinhilde D hulst, Tom Sterken [10] and others contrast the conversion efficiency between resistance as load and boost as load. It is concluded that the conversion efficiency of boost as load is $64 \%$, while only $40 \%$ resistance as load.

Double synchronous switch inductance circuit

Double synchronous switch inductance circuit can provide different load with a constant power output. Double synchronous switch inductance circuit principle as shown in figure 8, it includes two parts. Part one is the synchronous switch series inductance. It is composed of piezoelectric element, switch S1, inductance L1 and rectifier bridge. Part two is a Buck - Boost transform circuit. It is composed of the switch tube S2, inductance L2, diode and filter capacitor C2. The method to control the switch tube is different from traditional Buck - Boost conversion circuit.

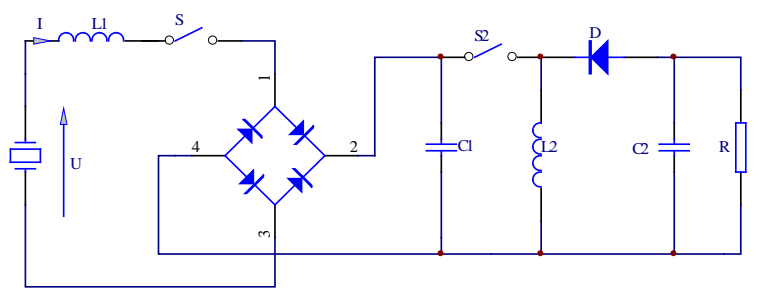

Figure 8 Double synchronous switch inductance circuit

\section{Conclusion}

This article simply introduces the research status of energy harvesting device, and sums up the energy collection of circuit design. Due to environmental vibration energy is relatively weak and irregular, the output of energy acquisition module cannot directly provide sufficient and stable electricity to the system. Therefore, we need to design suitable power management circuits to realize stable AC/DC conversion. In AC/DC conversion, the traditional bridge rectifier circuit is one of the typical applications, but its conversion efficiency is low. On this basis, the scholars put forward the optimization of the circuit structure to increase the conversion efficiency. This article makes the analysis of the relevant principles, and summarizes the advantages and disadvantages of all kinds of circuit existed. As you can see, high efficiency, low energy consumption, simple and reliable energy harvesting circuit is the main direction of future research. In addition to the structure, the energy collection circuit plays an important role in the influence factors of power. Combining the two, in order to achieve high performance, low power consumption, the intelligent vibrating energy collector is the micro energy development trend in the future. 


\section{Acknowledgments}

This project is supported by Natural Science Foundation of Jiangsu Province, China(Grant No. BK20131380) and Science and Technology Innovation Training Program of Nanjing University of Posts and Telecommunications (XZD2015025; XYB2015519) and Jiangsu Provincial Engineering Laboratory for RF Integration and Micropackaging.

\section{References}

[1] Suvradip Ghosh, Hsuan-Tsung Wang. A Circuit for Energy Harvesting Using On-Chip Solar Cells[J]. IEEE Transactions on power electronicsts, 2014:4658-4670.

[2] James Dicken, Paul D. Mitcheson, et al. Power-Extraction Circuits for Piezoelectric Energy Harvesters in Miniature and Low-Power Applications[J]. IEEE Transactions on power electronicsts, 2012, 11(27):4514-4529.

[3]Yuan Jiangbo, Shan Xiaobiao. Cantilever monocrystalline piezoelectric generator experiment [J]. Journal of optics, precision engineering, 2009 (5) : 1072-1077.

[4] Bai Fengxian, Bao Huayu, etc. In the piezoelectric vibration energy harvesting system of diode rectifier circuit equivalent RC model analysis [J]. Journal of power supply, 2014-5, 3:22-26.

[5]Lefeuvre E,Badel A,Richald c,et a1. Piezoelectric energy harvesting device optimization by synchmnous electric charge extraction[J]. Intelligent Material systems and stmctures, 2005, 16: 865-876.

[6]Lesfeuvre E, Badel A, Richard c, et a1. High-

performance piezoelectric vibration energy reclamation

[J].Proceeding the SPIE-The International Society for Optical Engineering, 2004, 5390:379-387.

[7] Hu Hongping, Wang Hairen. Piezoelectric can implement rectification interface on both ends of the nonlinear coupling experiment research [C]. Proceedings of the 2010 Symposium on Piezoelectricity,Acoustic Waves and Device Applications， 2010:424-427.

[8] Lesfeuvre G A, Ottman G K, Hofmann H F. Damping as a result of piezoelectric energy harvesting[J]. Joumal of sound and Vibration. 2004, 269: 99l-1001.

[9] Shafii A. Wahab, M. S. Bhuyan. Parametric Analysis of Boost Converter for Energy Harvesting using Piezoelectric for Micro Devices[J]. ICSE,2014, 525-528.

[10] Reinhilde D’hulst, Tom Sterken,et al. Power Processing Circuits for Piezoelectric Vibration-Based Energy Harvesters[J]. IEEE transactions on industrial electronics,2010,4170-4177. 